\title{
OPTIMAL CONTROL STRATEGY FOR MARINE SSP PODDED PROPULSION MOTOR BASED ON STRONG TRACKING-EPF
}

\author{
Wenlong Yao $\mathrm{Ya}^{\mathrm{a} b}$ \\ Yuan Liuc, \\ Honghai Sunc, \\ Guichen Zhangd, \\ Jundong Zhang, \\ Mingshun Zhou, \\ Ming Sun ${ }^{\mathrm{a}}$, \\ Dezhi Jianga, \\ a) Dept. of Marine Engineering, Qingdao Ocean Shipping Mariners College, \\ Qingdao, China \\ b) College of Marine Engineering, Dalian Maritime University, Dalian, China \\ c) Dept. of Naval Architecture \& Ocean Engineering, Qingdao Ocean \\ Shipping Mariners College, Qingdao, China \\ d) School of Shipbuilding Engineering, Journal of Harbin Engineering \\ University, Harbin, China
}

\begin{abstract}
Aiming at the non-linearity of state equation and observation equation of SSP (Siemen Schottel Propulsor) propulsion motor, an improved particle filter algorithm based on strong tracking extent Kalman filter (ST-EKF) was presented, and it was imported into the marine SSP propulsion motor control system. The strong tracking filter was used to update particles in the new algorithm and produce importance densities. As a result, the problems of particle degeneracy and sample impoverishment were ameliorated, the propulsion motor states and the rotor resistance were estimated simultaneously using strong track filter (STF), and the tracking ability of marine SSP propulsion motor control system was improved. Simulation result shown that the improved EPF algorithm was not only improving the prediction accuracy of the motor states and the rotor resistance, but also it can satisfy the requirement of navigation in harbor. It had the better accuracy than EPF algorithm.
\end{abstract}

Keywords: Marine electric propulsion, EPF, ST-EPF, SSP podded propulsion, Propulsion motor

\section{INTRODUCTION}

Marine podded propulsion is the new type of marine electrical propulsion system which has the advantages of simple structure, reliable operation and convenient maintenance, etc. But, the propulsion motor is more sensitive to load variation and external disturbance, such as thrust ripple, the cogging effect and nonlinear factors which have great influence to the end effect on tracking precision due to the marine podded propulsion system has no intermediate bearing and other transmission mechanism [3]. At present, the research of the propulsion motor control mainly focused on the areas of integral backstepping control [13], direct torque control [5], feedback linearization control [1], the sliding mode control [16],decoupling control [6], hybrid control method [2] and vector control [11] and so on, they have in common is that in view of the torque and flux linkage for independent control, so as to realize the linearization of the propulsion motor control.

In addition to utilize the advanced control method for the SSP propulsion motor speed sensorless control system, the accuracy of motor parameters is also the main factor which influence the control performance. However, the SSP propulsion motor in the process of running, parameters will be changed with the conditions and sea condition which shows the time-varying, when the fixed parameter model are utilized for calculating, the errors will be introduced inevitably, and the control performance will be degraded [15]. Therefore, the online identification of motor parameter has become the popular research, the motor parameters can be obtained by utilizing the constraints to simplify the model structure and reduce the measuring variables among the researches. Also the motor parameters can be identified by utilizing the 
model reference adaptive [4], observer estimation [7], artificial intelligence algorithm [8].

In addition, it is the key to the control system design for the marine electric propulsion system that the problems of the unknown rough sea conditions as well as the speed estimation in extremely low speed and zero speed [17]. Therefore, it need to be able to estimate the motor speed, at the same time, load torque, rotor flux motor parameter and the speed of the near zero for the parameters estimation method of the SSP control system. In paper [5], the direct torque control of permanent magnet synchronous motors without speed sensor is achieved through precisely estimating the stator flux linkage and rot or speed, and indirectly, the torque by means of the extended Kalman filter. Retaining the advantages of direct torque control method in rapid torque response and strong robustness, the system has significantly reduced ripples of flux linkage and torque. Besides, the effects of motor parameter variations and load disturbance are reduced.

Extended Kalman filter (EKF) is a novel filtering method of the nonlinear systems. However the traditional EKF method due to the partial linearization to transfer function and the measure function, can not adapt the serious non-linear situation; Moreover, derivate matrix of nonlinear function can not be carried out by utilizing the partial derivative [10], so its divergence has aroused much attention. The particle filter is an algorithm based on Monte Carlo methods. Its theory can be used in any non-linear and non-Gaussian state space models. R. V. Merwe brings forward the UPF (Unscented Particle Filter, UPF) algorithm [9] in which UKF (Unscented Kalman Filter, UKF) [12] was used to get more popular proposal distribution function so that the covariance of importance weights was reduced in the year of 2000. However, the EPF algorithm is realized by first order local linearization to the system equations based on Taylor series, the proposal distribution have bigger truncation errors, it results in the decrease of filtering precision, but it has the advantage of the relatively small amount of calculation. The UPF algorithm is realized by utilizing the UKF to get the importance of the particle filter function, although the accuracy can markedly be improved, but it has the disadvantages of too big amount of calculation and poor real-time performance.

The strong tracking extended Kalman particle filter algorithm (ST-EPF) is proposed in this article. The ST-EKF algorithm are utilized to update the particles in this algorithm. It is easy to cause the deviation of estimation even the phenomenon such as divergence, poor robustness, especially when it will lose the ability to mutation status tracking during it reaches steady state because of the controller word length and memory restrictions of the traditional EPF algorithm. The particle samples are processed by the ST-EKF algorithm in the ST-EPF algorithm, and the importance density are generated, the particle degradation and sample impoverishment problem can be relieved, their ability to mutation status tracking can also be improved and it can satisfy the mobility performance of the marine podded propulsion control system better.

\section{THE PARTICLE FILTER BASED ON ST-EKF}

\section{PRINCIPLE OF ST-EKF}

Time update equations

$$
P_{k+1, k}=\Phi_{k+1, k} P_{k} \Phi_{k+1, k}^{T}+Q_{k}
$$

Measurement updates equations:

$$
\begin{gathered}
K_{k+1}=P_{k+1, k} H_{k+1}^{T}\left[H_{k+1} P_{k+1, k} H_{k+1}^{T}+R_{k+1}\right]^{-1} \\
P_{k+1}=\left[I-K_{k+1} H_{k+1}\right] P_{k+1, k}
\end{gathered}
$$

The time-varying fading factor $\lambda_{\mathrm{k}+1}$ are introduced by the orthogonality principle in strong tracking filtering, the prediction error covariance $\mathrm{P}_{\mathrm{k}+1, \mathrm{k}}$ are adjusted, the gain matrix $\mathrm{K}_{\mathrm{k}+1}$ are controlled real-time, it makes the residual sequence of different time everywhere maintain orthogonality of orthogonal or approximate, the ability of the filter to track state changes is improved, it has a strong robustness and anti-interference ability, and the computational complexity is moderate. The equation (3)was simlified to the following form:

$$
P_{k+1, k}=L M D(k+1) \Phi_{k+1, k} \Phi_{k, k} \Phi_{k+1, k}^{T}+Q_{k}
$$

Where, $\operatorname{LMD}(k+1)=\operatorname{diag}\left[\lambda_{k+1}^{1}, \lambda_{k+1}^{2}, \cdots \lambda_{k+1}^{n}\right]$ is the timevarying fading matrix, $\lambda_{k+1}^{(i)} \geq 1, i=1,2, \cdots n$ is the time-varying fading factor $K_{k+1}=P_{k+1, k} H_{k+1}^{T}\left[H_{k+1} P_{k+1, k} H_{k+1}^{T}+R_{k+1}\right]^{-1}$. It is determined by orthogonal principle. To simplify the simulation which can be obtained by the method of literature, in the subprime:

$$
\begin{gathered}
\lambda_{k+1}^{(i)}=\left\{\begin{array}{cc}
\lambda_{k+1}, & \lambda_{k+1}>1 \\
1, & \lambda_{k+1} \leq 1
\end{array}\right. \\
\lambda_{k+1}=\operatorname{tr}\left[N_{k+1}\right] / \operatorname{tr}\left[M_{k+1}\right] \\
N_{k+1}=V_{k+1}-\beta R_{k}-H_{k} Q_{k} H_{k}^{T} \\
M_{k+1}=\Phi_{k} P_{k / k} \Phi_{k}^{T} H_{k}^{T} H_{k}
\end{gathered}
$$

Where, $\operatorname{tr}(\bullet)$ is the sum of the diagonal matrix, $\mathrm{N}_{\mathrm{k}+1}$ and $\mathrm{M}_{\mathrm{k}+1}$ are derived for the approximate orthogonal residual at different times, they have no specific physical meaning. $\mathrm{V}_{\mathrm{k}+1}$ is the output sequence of the mean square error matrix, it is calculated as follows:

$$
V_{k+1}=\left\{\begin{array}{cc}
\gamma_{1} \gamma_{1}^{T}, & k=0 \\
{\left[\rho V_{k}+\gamma_{k+1} \gamma_{k+1}^{T}\right] /(1+\rho),} & k \geq 1
\end{array}\right.
$$

Where, $0<\rho \leq 1$ is the residual sequence of forgetting factor, $\beta \geq 1$ is the selected weakening factor.

\section{THE IMPROVED PARTICLE FILTER ALGORITHM}

(1) Initialize with $(\mathrm{k}=0)$ : 
Draw the states $x_{0}{ }^{(i)}, p\left(x_{0}\right)$, assign the particle a weight

$$
\begin{gathered}
w_{0}{ }^{(i)}=1 / N \quad i=1,2 \cdots N \\
\bar{x}_{0}^{(i)}=W\left[x_{0}{ }^{(i)}\right] \\
P_{0}^{(i)}=E\left[\left(x_{0}{ }^{(i)}-\bar{x}_{0}{ }^{(i)}\right)\left(x_{0}{ }^{(i)}-\bar{x}_{0}{ }^{(i)}\right)^{T}\right]
\end{gathered}
$$

Where: $\mathrm{w}_{0}$ is the initial weights of support points, $\bar{x}_{0}$ is the initial value of the fixed state estimation, $\mathrm{P}_{0}$ is the initial value of matrix square-root of the state covariance.

(2) Importance Sampling( $\mathrm{k}=1,2, \ldots)$ :

We can get $\bar{x}_{k}^{(i)}, P_{k}^{(i)}$ by using the ST-EKF algorithm for each particle $x_{k-1}^{(i)}$ :

$$
\begin{gathered}
x_{k, k-1}^{(i)}=f\left(\bar{x}_{k-1}^{(i)}\right) \\
P_{k, k-1}^{(i)}=\lambda_{k}^{(i)} \Phi_{k}^{(i)} P_{k-1}^{(i)} \Phi_{k}^{(i) T}+Q_{k}^{(i)} \\
K_{k}^{(i)}=P_{k, k-1}^{(i)} H_{k}^{(i) T}\left(H_{k}^{(i)} P_{k, k-1}^{(i)} H_{k}^{(i) T}+R_{k}^{(i)}\right)^{-1} \\
\bar{x}_{k}^{(i)}=\bar{x}_{k, k-1}^{(i)}+K_{k}\left(z_{k}-h\left(x_{k, k-1}^{(i)}\right)\right) \\
\bar{P}_{k}^{(i)}=\left[I-K_{k} H_{k}^{(i)}\right] P_{k, k-1}^{(i)}
\end{gathered}
$$

Where: $z_{k}$ is the observed vectors that are dealt with by the ST-EKF, Q is the covariance matrix of the system noise series, $\mathrm{R}$ is the covariance matrix of the observed noise series which is dealt with by the ST-EKF.

We get proposal distribution function:

$$
q\left(x_{k}^{(i)} \mid x_{0: k-1}^{(i)}, z_{1: k}\right)=N\left(\bar{x}_{k}^{(i)}, P_{k}^{(i)}\right)
$$

The proposal distribution function makes use of the new observations, the sampling precision of particle was improved. We can get the new particles $\left\{x_{k}^{(i)}\right\}_{i=1}^{N}$ drawn from $x_{k}^{(i)} \otimes q\left(x_{k}^{(i)} \mid x_{0: k-1}^{(i)}, z_{1: k}\right)$

(3) Update and normalize the weights

Update:

$$
w_{k}^{(i)}=w_{k-1}^{(i)} \frac{p\left(z_{k} / x_{k}^{(i)}\right) p\left(x_{k}^{(i)} / x_{k-1}^{(i)}\right)}{q\left(x_{k}^{(i)} \mid x_{0: k-1}^{(i)}, z_{1: k}\right)}
$$

Normalize the weights

$$
\tilde{w}_{k}^{(i)}=w_{k}^{(i)} / \sum_{j=1}^{N} w_{k}^{(j)}
$$

(4) Resample

Resampling generates a new particle set by sampling from $\left\{\bar{x}_{k}^{(i)}, i=1,2 \cdots N\right\}$, with $j, \operatorname{Pr}\left\{x_{k}^{(i)}=\bar{x}_{k}^{(i)}\right\}=w_{k}^{(i)}$. Here $\mathrm{j}$ is the particle index after resampling.

The weight are reseted: $w_{k}^{(i)}=1 / N$.
(5) Update state

$$
x(k / k)=\frac{i}{N} \sum_{i=1}^{N} x_{k}^{(i)}
$$

It can be obtained from the step (2), $\bar{x}_{k}^{(i)}$ and $P_{k}^{(i)}$ are respectively mean and covariance through the calculation of ST-EKF. These handled observation vectors of particles reflect new prior with smaller covariance than former so that we can get more popular proposal distribution function, the covariance of importance weights has been reduced.

\section{MODEL ANALYSIS OF MARINE SSP PROPULSION MOTOR CONTROL SYSTEM}

The semi-submersible vessel „Kangshengkou” full electric propulsion system of Cosco is to be the research objection, the hardware of the control system was composed by the Siemens Simotion D, Sinamics, the upper computer and so on, the software of the control system contains Winccflexible, Step7, Scout and Matlab. This research was established in the basis of hardware-in-loop simulation of the SSP propulsion system which was based on the formal work [14], problem description is as follows:

Electromagnetic torque of the SSP propulsion is

$$
T_{e}=\frac{3 p}{2} \frac{\psi_{\mathrm{r}}^{2}}{R_{\mathrm{r}}} \omega_{\mathrm{s}}^{*}
$$

Where Te is electromagnetic torque, $\mathrm{P}$ is the number of magnetic poles, $\mathrm{Rr}$ is the rotor resistance, $\psi \mathrm{r}$ is the rotor flux valid values, $\frac{d \theta}{d t}=\omega$ is the given slip angular frequency, $\omega_{s}^{*}$ is the setting slip angular frequency. The rotor flux is the setting value actually ( $\mathrm{Li}$ et al., 2011), Te is proportional to the $T_{\mathrm{L}}=\operatorname{sgn}(\omega) \frac{K_{\mathrm{T}}}{4 \pi^{2}} \rho D^{5} \omega^{2}$.

The friction torque of SSP propulsion system $\mathrm{Tf}$ is

$$
T_{f}=\operatorname{sign}(\omega) T_{s}+k_{\omega} \omega
$$

Where $\omega=2 \pi n / 60, n$ is propeller speed, Ts is the static friction torque, $\mathrm{k}_{\omega}$ is the linear friction coefficient.

The motion equation of the SSP propulsion system is

$$
\begin{gathered}
\frac{d \omega}{d t}=\frac{1}{J}\left(T_{\mathrm{e}}-F \omega-T_{\mathrm{L}}\right) \\
\frac{d \theta}{d t}=\omega
\end{gathered}
$$

Where J is SSP moment of inertia, Tm is the load torque. The dynamic model of SSP propulsion system is

$$
\begin{gathered}
T_{\mathrm{p}}=K_{\mathrm{T}}(J) \cdot \rho D^{5} n|n| \\
F_{\mathrm{e}}=K_{\mathrm{F}}(J) \cdot \rho D^{4} n|n| \cdot(1-\tau) \\
P_{P}=2 \pi n T_{\mathrm{p}}=K_{\mathrm{T}}(J) \cdot 2 \pi \rho D^{5} n^{3}
\end{gathered}
$$

Where $T_{\mathrm{e}}\left(i_{\mathrm{a}}, i_{\mathrm{b}}, i_{\mathrm{c}}, \theta\right)=P \frac{\partial \mathbf{W}_{\mathrm{m}}(\psi, \theta)}{\partial \theta}, \mathrm{i}_{\mathrm{a}}$ is thrust deduction coefficient, $\mathrm{i}_{\mathrm{b}}$ is wake fraction, the value can be obtained 
by ship's manual, Tp is propeller torque, $\mathrm{Fe}$ is propeller effective thrust, $\rho$ is density of water, $D$ is propeller diameter, $\mathrm{KT}(\mathrm{J})$ is torque coefficient of dimensionless, $\mathrm{KF}(\mathrm{J})$ is the thrust coefficient of dimensionless, $\mathrm{KT}(\mathrm{J})$ and $\mathrm{KF}(\mathrm{J})$ are the function of J, they can be obtained by the propeller working characteristic curve. During stable sailing in the sea, the ratio of propeller is a constant value, it shows that propeller will work on the speed ratio of the characteristic curve.

\section{SIMULATION AND RESULTS}

The marine SSP propulsion motor control system was established under the Matlab/Simulinkenvironment, the propeller model was realized by utilizing the $\mathrm{S}$-function. The simulation parameter of propulsion motor was based on "Kangshengkou", as follows: power rating $=4700 \mathrm{~kW}$, rated voltage $=660 \mathrm{~V}$, rated speed $=155 \mathrm{r} / \mathrm{min}$, rotor permanent magnet flux linkage $=4.55 \mathrm{~Wb}$, rated torque $=1185 \mathrm{KN} \cdot \mathrm{m}$, stator resistance $=1.632 \mathrm{~m}$,number of motor pole $=8$, inductance of $\mathrm{d}$ axis $=0.25 \mathrm{mH}$, inductance of $\mathrm{q}$ axis $=0.47 \mathrm{mH}$.

When ship navigation in harbor at $130 \mathrm{r} / \mathrm{min}$, it needs to manoeuvre the vehicle frequently, the simulation analysis was carried out on this working condition. The simulation time is $1 \mathrm{~s}$, the initial speed is $130 \mathrm{r} / \mathrm{min}$, at the beginning of the simulation, the motor load torque is $240 \mathrm{KN} \cdot \mathrm{m}$, at the time of $0.5 \mathrm{~s}$ the speed was changed to $110 \mathrm{r} / \mathrm{min}$. At the same time the sea condition suddenly changes, which at the beginning, the motor load torque is $240 \mathrm{KN} \cdot \mathrm{m}$, at the time of $0.36 \mathrm{~s}$ propeller load torque changes to $220 \mathrm{KN} \cdot \mathrm{m}$. The simulation results about the control method of SSP propulsion motor based on vector control with EPF and ST-EPF composite algorithm are shown as Figure 1- Figure 6.

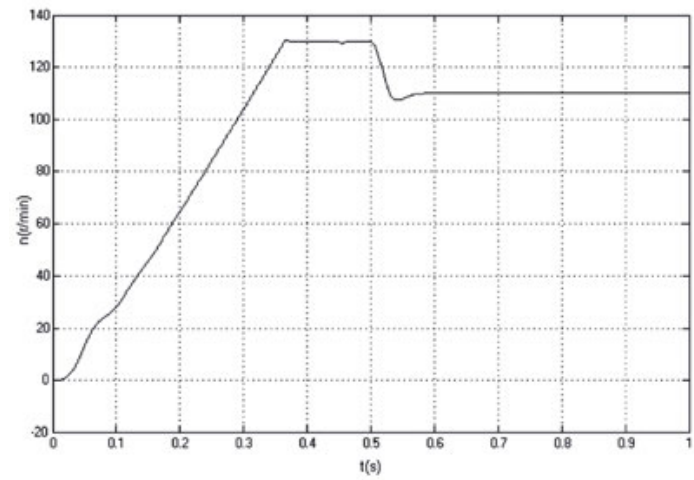

Fig. 1. Speed curve based on EPF

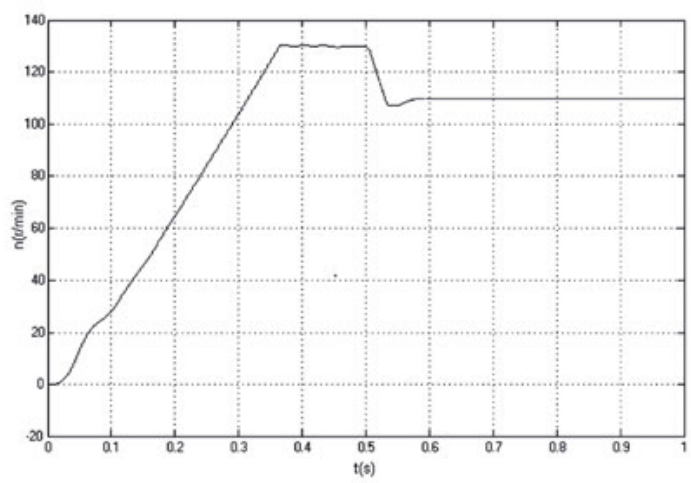

Fig. 2. Speed curve based on ST-EPF

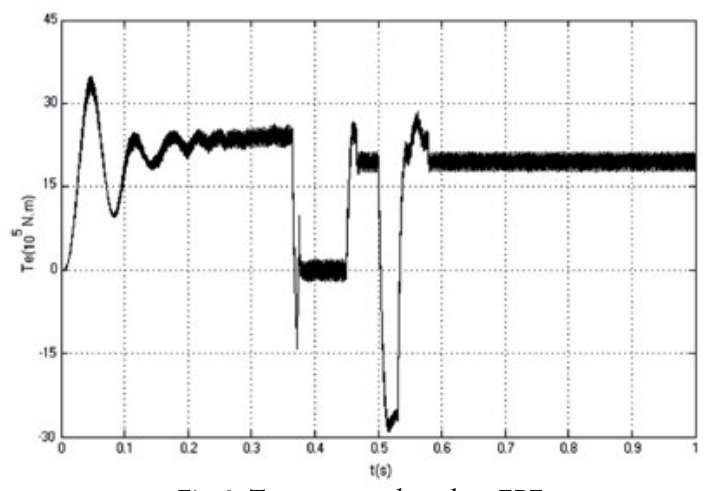

Fig. 3. Torque curve based on EPF

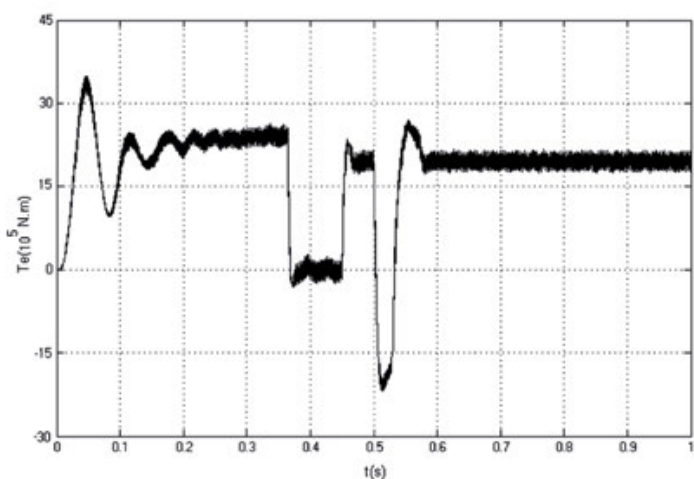

Fig. 4. Torque curve based on ST-EPF

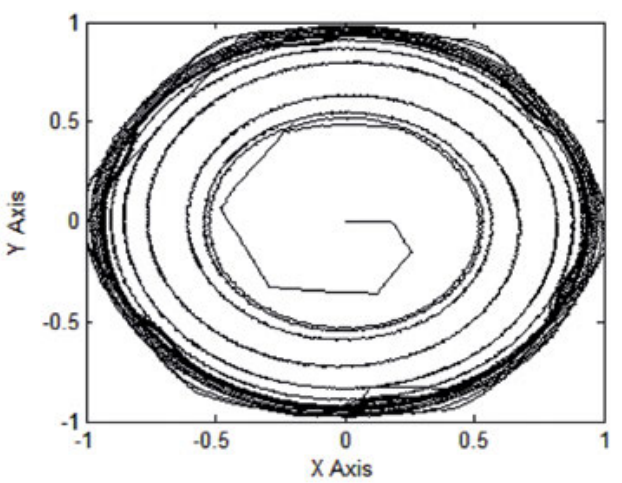

Fig. 5. Stator flux based on EPF

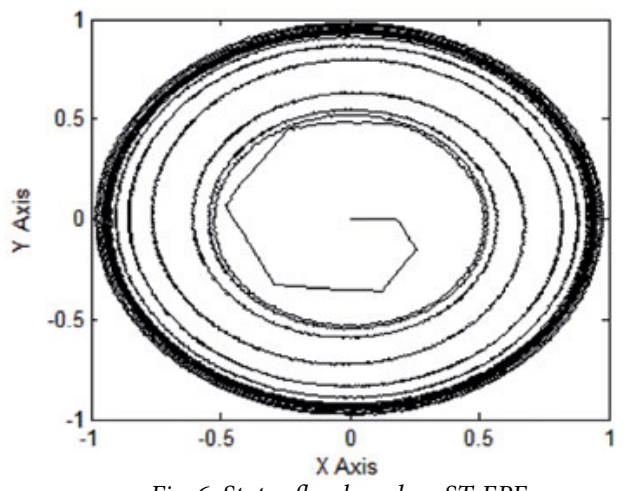

Fig. 6. Stator flux based on ST-EPF

The simulation results show that: When ship navigation in harbor and with the rough sea, under the two control modes, speed up smoothly, and the speed changes slightly after the torque of the propeller load changes but back then. The system reaches the given speed $130 \mathrm{r} / \mathrm{min}$ after $0.36 \mathrm{~s}$ and propeller 
load changes in $0.36 \mathrm{~s}$, the controller, torque and current have a corresponding response by the ST-EPF. It can be shown that the SSP propulsion system based on ST-EPF composite control has the lower speed fluctuation, smaller steady state operating static error and better dynamic response ability than which based on EPF.

\section{CONCLUSIONS}

In this paper the ST-EPF control algorithm was introduced into the sensorless vector control of marine propulsion motor control system, the orthogonality principle was introduced to the tracking process of control system, the gain matrix was controlled real-time, the track state mutation ability of the filter was improved. The simulation results show that the ST-EPF observer for the control system has good robustness, especially to the changes of the system parameter, the adaptability of measurement noise and system noise are superior to the EPF observer, the EPF observer error problem of poor robustness of the model was better solved, the marine podded propulsion motor flux and speed can be estimated accurately.

\section{ACKNOWLEDGMENTS}

This work is supported by Shinan District Science and Technology Development Funds of Qingdao City under Grant 2012-5-004-ZH and 2012-5-005-ZH, the Shandong Province Higher Educational Science and Technology Program under Grant J13LN72, National Natural Science Foundation (NNSF) of China under Grant 51179102, Ministry of industry and information Technology of China branch project under Grant 201301188, Science and Technology Program of COSCO under Grant 2012-1-H-004 and 2014-1-H-005.

\section{REFERENCES}

1. Angelo C, Bossio G, Garcia G, et al.,2006. Speed control of PMSMs with interconnection an damping assignment or feedback linearization comments about their performance. Industrial Electronics. Montreal, 2182-2187.

2. Cetin E, Oguz U,2008. A hybrid controller for the speed of a permanent magnet synchronous motor drive [J]. Control Engineering Practice,16, 260-270.

3. Islam M F, Veitch B, Liu P, et al., 2010. Gap Effect on Performance of Podded Propulsors in Straight-Ahead and Azimuthing Conditions. Marine Technology, 47,47-58.

4. Ji Feng,Fu Lijun, Ye Zhihao,2011. Study on Vector Control for Vessel Electric Propulsion. Journal of wuhan university of science and technology,35,361-364

5. LI Bingqiang, LIN Hui,2011. Direct Control of Current Vector for Surface-mounted Permanent Magnet Synchronous Motor. Proceedings of the CSEE,31,288-294
6. LI Liang-liang, HE Yong,YE Hai-xiang, 2011. Simulation of Permanent Magnet Synchronous Motor Vector Control Based on ITAE Optimization. Electric Machines \& Control Application, 38, 31-45

7. Li Zhongbing,Zhang Huanren,2011. Extended Kalman Filter Enhanced Ship Electrical Propulsion System, Navigation of China, 34, 45-50

8. Lu Wenbin Yao Wenxi Lü Zhengyu,2013. Speed Sensorless Vector Control with Improved Closed-Loop Flux Observer for Induction Machines. Transactions of China Electro Technical Society,28, 148-153.

9. R.V.Merwe,A.Douvet,N.De.Freitas et al,2000. The unscented particle filter.Technical Report CUED/F@ INPENG/ TR380:Cambridge University Engi -neering Department.

10. Sui Shu-lin, Yao Wen-long,2008. Spacecraft of autonomous optical navigation Based on wavelet-UPF. Systems Engineering and Elctronics. 8,1519-1522

11. Suman Maiti, Chandan Chakraborty, Sabyasachi Sengupta, 2009. Simulation studies on model reference adaptive controller based speed estimation technique for the vector controlled permanent magnet synchronous motor drive. Simulation Modelling Practice and Theory,7, 585-596.

12. Van Dyke M C, Schwartz J L , Hall C D,2004. Unscented Kalman filtering for spacecraft attitude state and parameter estimation. http: www. Space-flight org/AAS_ meetings/ 2004_ winter /w2004-program.pdf, 2004-0310 .

13. WANG Li-peng, ZHANG Hua-guang,LIU Xiuchong,2012. Integral backstepping controller in the sensorless vector-control system for permanent magnet synchronous motor. Control Theory \& Applications, 29,199-204

14. Wenlong Yao, Yuan Liu, Jundong Zhang, Sun Ming, Zhang Gui-chen and Wei shao,2013. Design of Vector Control based on MFAC for SSP Podded Propulsion, Proc. of the Int. Conf. on Robotics and Biomimetics (ROBIO 2013), $12,2418-2423$

15. Wenlong Yao, Jundong Zhang,Ronghu Chi, Zhang Gui-chen. Model-free adaptive vector control of ship podded SSP propulsion motor, Journal of Traffic and Transportation Engineering, 2014,14(6): 59-66.

16. YU Ming, CONG Shuang, XU Juan,2008. Design of nonlinear motor adaptive fuzzy sliding mode controller based on GA, Journal of System Simulation. 20, 31413145 . 
17. ZHANG Guichen, MA Jie, 2010. Study and Application of Podded Electric Propulsion System Based on SIMOTION.

Ship Building of China, 51,45-50.

\title{
CONTACT WITH AUTHOR
}

\author{
Wenlong Yao \\ yaowenlong@dlmu.edu.cn \\ tel:+86 53285752355 \\ fax:+86 53285752355 \\ Mingshun Zhou \\ Ming Sun \\ Dezhi Jiang
}

Dept. of Marine Engineering,

Qingdao Ocean Shipping Mariners College

Qingdao, China

\author{
Wenlong Yao \\ Jundong Zhang \\ College of Marine Engineering \\ Dalian Maritime University \\ Dalian, China
}

Yuan Liu

Honghai Sun

Dept. of Naval Architecture \& Ocean Engineering

Qingdao Ocean Shipping Mariners College

Qingdao, China

Guichen Zhangd

School of Shipbuilding Engineering Journal of Harbin Engineering University

Harbin, China 